\title{
Молекулярно-пучковая эпитаксия двухмерных слоев GaSe на подложках GaAs(001) и GaAs(112): структурные и оптические свойства
}

\author{
(C) С.В. Сорокин, П.С. Авдиенко, И.В. Седова, Д.А. Кириленко, М.А. Яговкина, \\ А.Н. Смирнов, В.Ю. Давыдов, С.В. Иванов
}

Физико-технический институт им. А.Ф. Иоффе Российской академии наук, 194021 Санкт-Петербург, Россия

E-mail: sorokin@beam.ioffe.ru

Поступила в Редакцию 14 марта 2019 г.

В окончательной редакции 1 апреля 2019 г.

Принята к публикации 1 апреля 2019 г.

Представлены результаты исследования структурных и оптических свойств двумерных (2D) слоев $\mathrm{GaSe}$, выращенных методом молекулярно-пучковой эпитаксии на подложках $\operatorname{GaAs}(001)$ и $\operatorname{GaAs}(112)$ при использовании источника $\mathrm{Se}$ с высокотемпературным разложителем и клапаном. Определено влияние параметров роста (температуры подложки, интенсивности потока атомов $\mathrm{Ga}$, отношения падающих потоков $\mathrm{Se} / \mathrm{Ga})$ на морфологию поверхности выращенных слоев. С помощью методов просвечивающей электронной микроскопии, электронной дифракции и спектроскопии комбинационного рассеяния света показано, что слои GaSe соответствуют политипу $\gamma$-GaSe. Методом рентгеновской дифрактометрии установлено наличие включений фазы $\alpha-\mathrm{Ga}_{2} \mathrm{Se}_{3}$ в слоях $\mathrm{GaSe}$, выращенных в условиях сильного обогащения поверхности роста селеном.

Ключевые слова: GaSe, слоистые полупроводники, молекулярно-пучковая эпитаксия, структурные свойства.

DOI: $10.21883 /$ FTP.2019.08.48010.9103

\section{1. Введение}

Материалы с экстремальной двумерностью $\left(\mathrm{MoS}_{2}\right.$, $\mathrm{GaTe}, \mathrm{GaSe}, \mathrm{InSe}$ и т.д.) представляют собой слоистые полупроводниковые соединения, обладающие уникальными структурными, электрическими и оптическими свойствами. В отличие от наиболее исследованного слоистого полупроводника $\mathrm{MoS}_{2}$, ширина запрещенной зоны GaSe лежит в видимом диапазоне спектра ( 2 эВ при $300 \mathrm{~K})$, что делает его одним из наиболее перспективных двумерных (2D) материалов для оптоэлектронных применений. Объемные кристаллы GaSe состоят из вертикально упорядоченных слоев толщиной $\sim 0.8 \mathrm{HM}$, которые удерживаются вместе слабыми силами Ван-дерВаальса, при этом каждый слой состоит из четырех ковалентно-связанных атомных слоев $\mathrm{Se}-\mathrm{Ga}-\mathrm{Ga}-\mathrm{Se}$ (так называемый тетраслой). Различные варианты упаковки слоев приводят к возникновению разных объемных кристаллических структур $(\beta, \varepsilon, \gamma, \delta)[1]$, при этом влияние политипов на структурные и оптические свойства незначительно. В атмосферных условиях поверхность пленок GaSe окисляется, что может приводить к деградации оптических свойств [2]. Тем не менее недавно было показано, что пассивация поверхности выращенных слоев $\mathrm{GaSe}$ слоем $\mathrm{Al}_{2} \mathrm{O}_{3}$ толщиной $50 \mathrm{Hм}$ позволяет в существенной степени подавить процесс окисления [3]. В настоящее время тонкие пленки GaSe получают преимущественно методом механической эксфолиации, что существенно ограничивает возможность коммерческого использования данного материала для приборных применений. В этой связи актуальным является развитие эпитаксиальных методов выращивания GaSe c целью формирования высококачественных 2D кристаллов большой площади с высокой степенью однородности. Несмотря на значительный рост количества публикаций по 2D полупроводниковым соединениям в последние годы [4], работ, посвященных технологии выращивания методом молекулярно-пучковой эпитаксии (МПЭ) слоев и $\mathrm{A}^{\mathrm{III}} \mathrm{B}^{\mathrm{VI}}$-гетероструктур на основе $\mathrm{GaSe}$, весьма немного, причем значительная их часть была опубликована в 90-е годы прошлого века [5-8].

Вместе с тем использование МПЭ в качестве основного технологического метода выращивания полупроводниковых 2D кристаллов и гетероструктур на их основе в режиме эпитаксии Ван-дер-Ваальса [9] обладает рядом преимуществ: легкость прецизионного формирования слоев заданной толщины, возможность достижения атомарной резкости гетерограниц и использование подложек без учета степени рассогласования постоянных кристаллических решеток.

Настоящая работа посвящена детальному исследованию структурных и оптических свойств эпитаксиальных слоев GaSe, выращиваемых методом МПЭ на подложкаx GaAs различной ориентации в условиях широкого варьирования параметров роста.

\section{2. Эксперимент}

Слои GaSe выращивались в двухкамерной установке МПЭ (SemiTEq, Россия) на подложках $\mathrm{GaAs}(001)$ 
„epi-ready“ и химически подготовленных подложках $\mathrm{GaAs}(112)$. В качестве источников молекулярных пучков были использованы стандартный элементный источник $\mathrm{Ga}$ и клапанный источник Se c разложителем (Veeco, США). Отжиг подложек проводился в условиях сверхвысокого вакуума в отсутствие потока As при температуре $T_{s} \approx 580^{\circ} \mathrm{C}$. Нагрев подложки осуществлялся радиационно-бесконтактным способом. Изменение потока атомов $\mathrm{Se}$ контролировалось посредством изменения температуры зоны „bulk“ источника Se. Температура зоны разложения (cracking zone) $T_{\mathrm{Se}}(\mathrm{cr})=500^{\circ} \mathrm{C}$ и положение клапана источника $\mathrm{Se}$ оставались неизменными от процесса к процессу. Варьируемыми параметрами при росте $\mathrm{GaSe}$ являлись температура подложки $\left(T_{s}=400-500^{\circ} \mathrm{C}\right)$, температура источника $\mathrm{Ga}$ $\left(T_{\mathrm{Ga}}\right)$, определяющая интенсивность потока атомов $\mathrm{Ga}$, и отношение потоков $\mathrm{Se}$ и $\mathrm{Ga} R=P_{\mathrm{Se}}(\mathrm{BEP}) / P_{\mathrm{Ga}}(\mathrm{BEP})$, $8<R<48$ (BEP - beam equivalent pressure, эквивалентное давление пучка, измеряемое ионизационным датчиком Байярда-Альперта в позиции подложки). Рост инициировался одновременным открытием потоков $\mathrm{Ga}$ и Se на поверхность GaAs. Процесс роста GaSe контролировался in situ при помощи метода дифракции быстрых электронов на отражение (ДБЭО).

Для исследования морфологии поверхности и определения толщины выращенных слоев $\mathrm{GaSe}$ использовался метод растровой электронной микроскопии (РЭМ). Структурные характеристики образцов исследовались методом рентгеновской дифрактометрии (РД) с использованием порошкового рентгеновского дифрактометра D2 Phaser (Bruker, Германия) и просвечивающей электронной микроскопии (ПЭМ) с использованием микроскопа Jeol JEM-2100F (ускоряющее напряжение 200 кB, разрешение по точкам 0.19 нм). Образцы в геометрии поперечного сечения готовились по стандартной методике: механическая шлифовка с последующим утончением посредством травления ионами $\mathrm{Ar}^{+}, 4$ кэВ.

Слои $\mathrm{GaSe}$ были исследованы также методом спектроскопии комбинационного рассеяния света (КРС). Измерения КРС выполнялись при комнатной температуре на спектрометрической установке Т64000, укомплектованной конфокальным микроскопом, что позволяло исследовать области диаметром $\sim 1$ мкм. В качестве источника возбуждения использовался твердотельный $\mathrm{Nd}$ : YAG-лазер (длина волны возбуждени $\lambda_{\mathrm{ex}}=532 \mathrm{Hм}$ ), мощность лазерного излучения на образце составляла $\sim 80$ мкВт.

\section{3. Результаты и обсуждение}

Эпитаксия по механизму Ван-дер-Ваальса при МПЭ $\mathrm{GaSe}$ на подложках $\mathrm{GaAs}(001)$ реализуется, как было показано ранее, при температурах подложки $T_{s} \approx 400^{\circ} \mathrm{C}$, при этом ось с выращиваемого слоя GaSe ориентирована в направлении нормали к поверхности подложки [5]. При более высокой температуре роста $\left(T_{s}>500^{\circ} \mathrm{C}\right)$ зарождение $\mathrm{GaSe}$ на поверхности $\mathrm{GaAs}(001)$ происходит через формирование переходных субмонослоев с образованием химических связей с подложкой $[5,10]$. Поэтому для получения высокотемпературных слоев $\mathrm{GaSe}$ с ориентированной в выделенном направлении осью с предлагалось использовать подложки $\mathrm{GaAs}(001)$, разориентированные на угол $2-5^{\circ}$ в направлении $[\overline{1} 10][7,10]$.

Известно, что одним из основных факторов, определяющих свойства выращенных слоев $\mathrm{GaSe}$, является температура эпитаксиального роста. Однако при малой интенсивности потока $\mathrm{Ga}$ (соответствующей скорости осаждения GaSe $\sim 2$ нм/мин при $T_{s} \approx 400^{\circ} \mathrm{C}$ ) и высокой температуре подложки $\left(T_{s}>500^{\circ} \mathrm{C}\right)$ наблюдается переиспарение GaSe с поверхности роста [10]. Другими словами, высокое значение $T_{s}$ и малые значения интенсивности потока атомов $\mathrm{Ga}$ являются взаимоисключающими факторами при МПЭ GaSe на подложках $\operatorname{GaAs}(001)$.

Ранее было показано, что оптимальными условиями при МПЭ $\mathrm{GaSe}$ на подложках $\mathrm{GaAs}(001)$ являются условия, близкие к стехиометрическому соотношению адсорбированных адатомов $\mathrm{Ga}$ и $\mathrm{Se}$ на поверхности роста. При использовании стандартных элементных источников $\mathrm{Ga}$ и $\mathrm{Se}$ эти условия соответствуют отношению эквивалентных давлений в падающих потоках, измеряемых ионизационным датчиком Байярда-Альперта, $P_{\mathrm{Se}} / P_{\mathrm{Ga}}(\mathrm{BEP}) \approx 4$ при $T_{s} \approx 500-540^{\circ} \mathrm{C}[5,7]$ и $\sim 2.4[5]$ при $T_{s}=350^{\circ} \mathrm{C}$. При использовании в качестве источника $\mathrm{Se}$ клапанного источника с температурой зоны разложения $T_{\mathrm{Se}}(\mathrm{cr})=950^{\circ} \mathrm{C}$ стехиометрии на поверхности роста соответствуют отношения потоков $P_{\mathrm{Se}} / P_{\mathrm{Ga}}(\mathrm{BEP}) \approx 50$ при $T_{s}=350^{\circ} \mathrm{C}, \sim 100$ при $T_{s}=400^{\circ} \mathrm{C}$ и $\sim 200$ при $T_{s}=450^{\circ} \mathrm{C}[11,12]$. Столь высокие значения отношений потоков $\mathrm{Se} / \mathrm{Ga}$ обусловлены как низкой способностью к встраиванию атомов и молекул селена, разогретых до высокой температуры в зоне разложения источника $\mathrm{Se}$, так и сильной зависимостью коэффициента встраивания Se от температуры подложки [13].

Учитывая, что различие отношений падающих потоков $\mathrm{Se}$ и $\mathrm{Zn} P_{\mathrm{Se}} / P_{\mathrm{Zn}}(\mathrm{BEP})$, соответствующих стехиометрии на поверхности роста при МПЭ $\mathrm{ZnSe}$, при использовании эффузионного источника $\mathrm{Se}$ и клапанного источника с $T_{\mathrm{Se}}(\mathrm{cr})=500^{\circ} \mathrm{C}$ составляет $\sim 2.5$ при $T_{s}=300-330^{\circ} \mathrm{C} \quad[13]$, и используя данные работы [11], можно предположить, что при МПЭ GaSe при $T_{\mathrm{Se}}(\mathrm{cr})=500^{\circ} \mathrm{C}$ стехиометрическим условиям соответствует отношение падающих потоков $P_{\mathrm{Se}} / P_{\mathrm{Ga}}(\mathrm{BEP}) \approx 12$ при $T_{s} \approx 400^{\circ} \mathrm{C}$ и $\sim 16$ при $T_{s} \approx 450^{\circ} \mathrm{C}$.

Корректность данной оценки была подтверждена нами наблюдением образования капель $\mathrm{Ga}$ на поверхности роста при $T_{s} \approx 400^{\circ} \mathrm{C}$ и отношении потоков $P_{\mathrm{Se}} / P_{\mathrm{Ga}}(\mathrm{BEP}) \approx 8$, а также при $T_{s} \approx 500^{\circ} \mathrm{C}$ и отношении потоков $\sim 17$. Результирующая феноменологическая зависимость отношения эквивалентных давлений пучка $P_{\mathrm{Se}} / P_{\mathrm{Ga}}$, соответствующих стехиометрии на поверхности роста при различных $T_{s}$, от температуры зоны раз- 
Параметры выращенных слоев GaSe

\begin{tabular}{|c|c|c|c|c|c|c|}
\hline Номер образца & Тип подложки & $T_{\mathrm{Se}}(\mathrm{cr}),{ }^{\circ} \mathrm{C}$ & $T_{s},{ }^{\circ} \mathrm{C}$ & $\begin{array}{c}\text { Отношение потоков } \\
P_{\mathrm{Se}} / P_{\mathrm{Ga}}(\mathrm{BEP})\end{array}$ & $\begin{array}{c}\text { Толщина слоя, } \\
\text { нм }\end{array}$ & $\begin{array}{c}\text { Скорость роста, } \\
\text { нм/мин }\end{array}$ \\
\hline \#A & \multirow{2}{*}{$\operatorname{GaAs}(001)$} & \multirow{6}{*}{500} & \multirow{4}{*}{400} & 48 & 270 & 1.13 \\
\hline$\# \mathrm{~B}$ & & & & 32 & 200 & 1.3 \\
\hline$\# \mathrm{C}$ & \multirow{2}{*}{$\mathrm{GaAs}(112)$} & & & 20 & 83 & 1.4 \\
\hline \#D & & & & 14 & 86 & 1.45 \\
\hline$\# \mathrm{E}$ & \multirow{2}{*}{$\operatorname{GaAs}(001)$} & & 500 & $17-20$ & 45 & $\sim 4$ \\
\hline$\# \mathrm{~F}$ & & & 400 & 12 & 300 & $\sim 5$ \\
\hline
\end{tabular}

ложения клапанного источника $\mathrm{Se}$ приведена на рис. 1. Из этой зависимости прямо следует, что управлять стехиометрией на поверхности роста при фиксированной $T_{s}$ можно как за счет регулировки открытия клапана источника Se, так и за счет вариации температуры зоны разложения $T_{\mathrm{Se}}(\mathrm{cr})$. В последнем случае изменение соотношения адатомов $\mathrm{Se}$ и $\mathrm{Ga}$ на поверхности роста определяется изменением коэффициента встраивания $\mathrm{Se}$ [13].

Влияние отношения падающих потоков $P_{\mathrm{Se}} / P_{\mathrm{Ga}}$ на морфологию поверхности показано на рис. 2, где представлены изображения поверхности эпитаксиальных слоев $\mathrm{GaSe}$, полученные методом РЭМ. Для всех приведенных образцов (за исключением \#Е) температура роста составила $T_{s} \approx 400^{\circ} \mathrm{C}$; скорость роста варьировалась в диапазоне $1-5 \mathrm{Hм} /$ мин; толщины слоев GaSe,

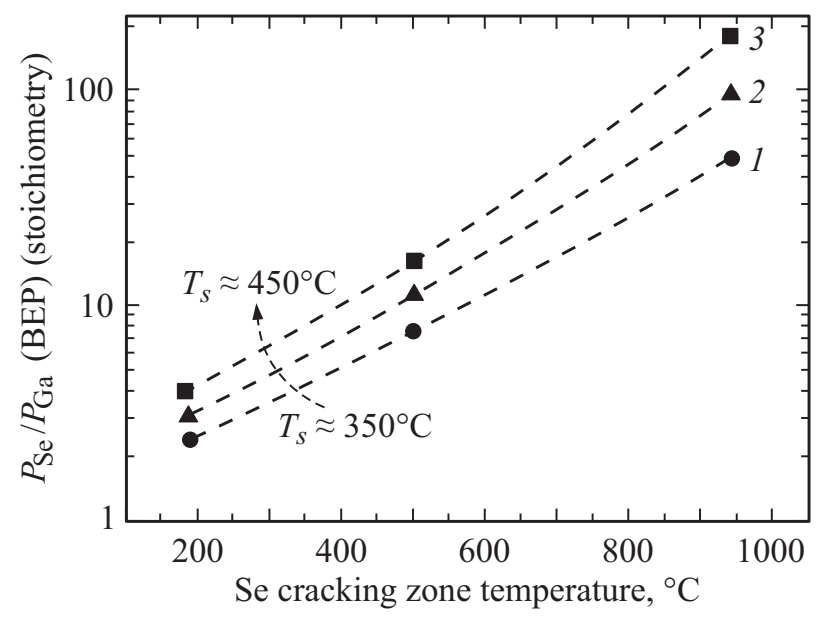

Рис. 1. Зависимость отношения $R=P_{\mathrm{Se}} / P_{\mathrm{Ga}}(\mathrm{BEP})$, соответствующего стехиометрии на поверхности роста, от температуры зоны разложения клапанного источника Se для температур подложки $T_{s}=350(1), 400(2), 450^{\circ} \mathrm{C}(3)$. Штриховые линии проведены для наглядности. При построении зависимости использованы экспериментальные данные из работ $[5,7]$ для стандартного элементного источника $\mathrm{Se}\left(T_{\mathrm{Se}} \approx 200^{\circ} \mathrm{C}\right)$, из работ $[11,12]$ для клапанного источника Se с температурой зоны разложения $T_{\mathrm{Se}}(\mathrm{cr})=950^{\circ} \mathrm{C}$ и оценочные данные настоящей работы для клапанного источника $\mathrm{Se}$ с температурой зоны разложения $T_{\mathrm{Se}}(\mathrm{cr})=500^{\circ} \mathrm{C}$. согласно данным измерений методом РЭМ в геометрии поперечного сечения, составили 45-300 нм. Основные параметры слоев $\mathrm{GaSe}$, морфология поверхности которых представлена на рис. 2, приведены в таблице.

Из анализа изображений поверхности слоев GaSe, выращенных при близких значениях скоростей роста $v_{\mathrm{GaSe}} \approx 1.1-1.45$ нм/мин (образцы \#А, \#В, \#С, \#D) следует, что при приближении условий МПЭ к стехиометрическим, т.е. при уменьшении отношения падающих потоков $\mathrm{Se} / \mathrm{Ga}$, количество так называемых нанопластинок (nanoplatelets) на поверхности GaSe существенно уменьшается. Поверхность слоя становится более планарной.

Подобную закономерность наблюдали ранее при MПЭ GaSe на подложках GaN [12]. Для образца \#D, выращенного на подложке $\mathrm{GaAs}(112)$ при отношении потоков $P_{\mathrm{Se}} / P_{\mathrm{Ga}} \approx 14$, нанопластинки на поверхности роста практически отсутствуют. Планарный рост слоя \#D подтверждается данными ДБЭО. Четкие полосковые рефлексы с отсутствием точек и концентрических колец на дифракционной картине регистрировались на протяжении всего роста структуры. При увеличении температуры эпитаксиального роста с $T_{s}=400^{\circ} \mathrm{C}$ до $500^{\circ} \mathrm{C}$ поверхность становится более шероховатой, что хорошо согласуется с данными работ $[5,12]$.

Третьим ключевым фактором, оказывающим влияние на морфологию поверхности слоев $\mathrm{GaSe}$, является скорость эпитаксиального роста, определяемая потоком атомов Ga. При большой интенсивности потока атомов $\mathrm{Ga}$ (соответствующей скорости роста $\left.v_{\mathrm{GaSe}} \approx 4 \mathrm{Hм} / \mathrm{Mин}\right)$ и низкой температуре роста $\left(T_{s} \approx 400^{\circ} \mathrm{C}\right)$ подвижности атомов галлия, по-видимому, недостаточно для формирования слоя с планарной морфологией (структура \#F, рис. $2, f)$. Необходимость малой скорости осаждения для формирования планарных слоев GaSe подтверждается, например, данными [14], где авторы наблюдали осцилляции ДБЭО при МПЭ $\mathrm{GaSe}$ на подложках $\mathrm{Si}(001)$ и слюды со скоростью роста в диапазоне $0.3-0.8$ нм/мин.

Исследование характеристик выращенных образцов $\mathrm{GaSe}$ проводилось методами КРС, РД и ПЭМ. На рис. 3, а приведено ПЭМ-изображение в геометрии поперечного сечения слоя \#В, выращенного на подложке 


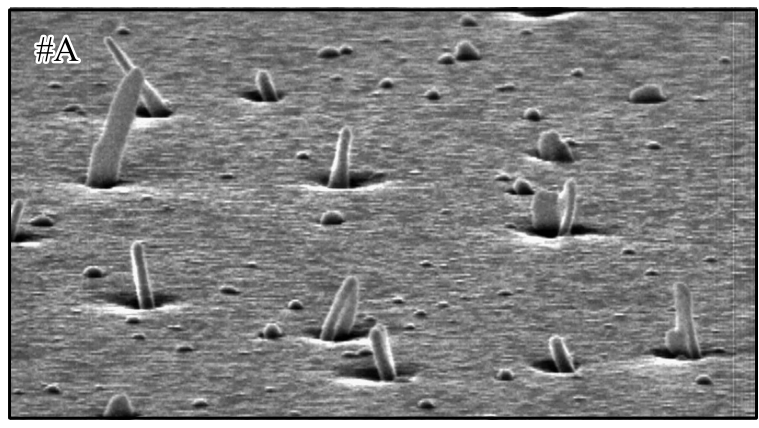

$20 \mathrm{kV}$

$1 \mu \mathrm{m} \longrightarrow$

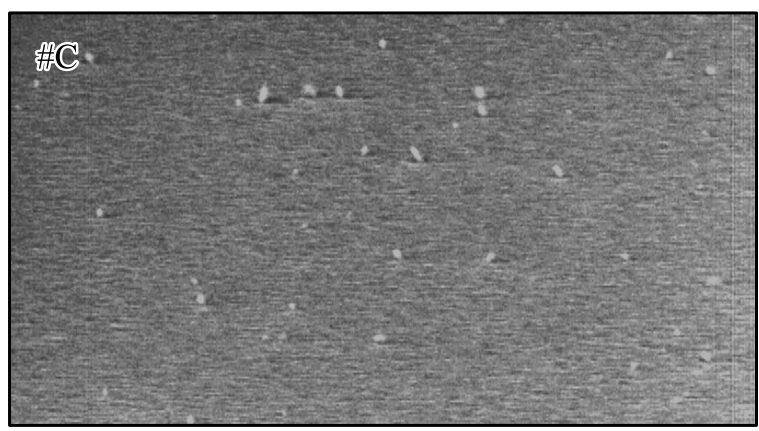

$20 \mathrm{kV}$

$1 \mu \mathrm{m}$

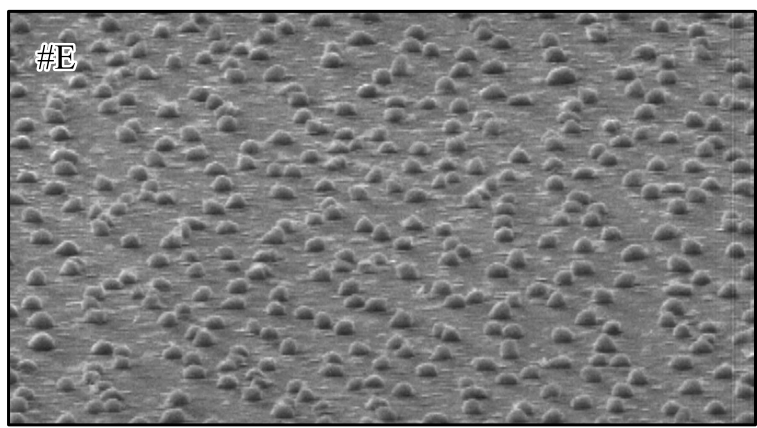

$20 \mathrm{kV}$

$1 \mu \mathrm{m}$ $a$

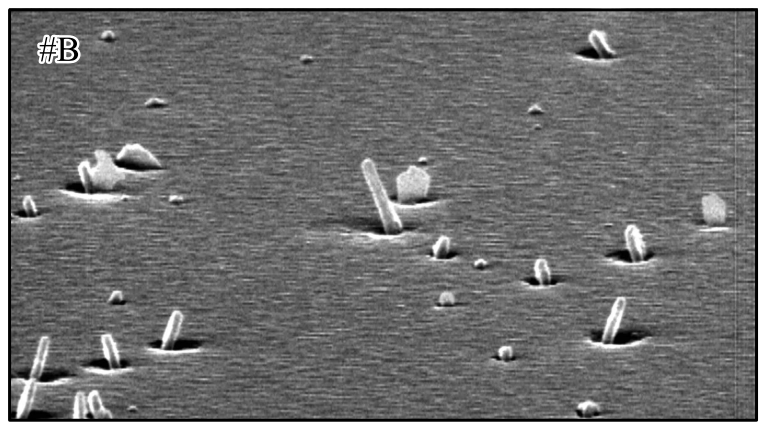

$20 \mathrm{kV}$

$1 \mu \mathrm{m} \longrightarrow$

$c$

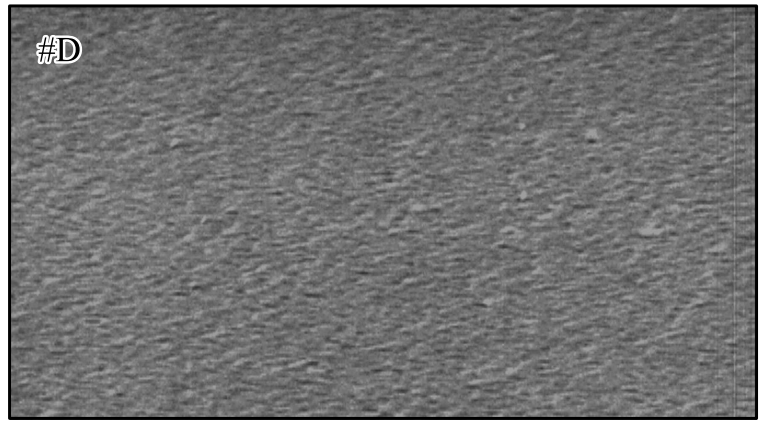

$20 \mathrm{kV}$

$1 \mu \mathrm{m}-$

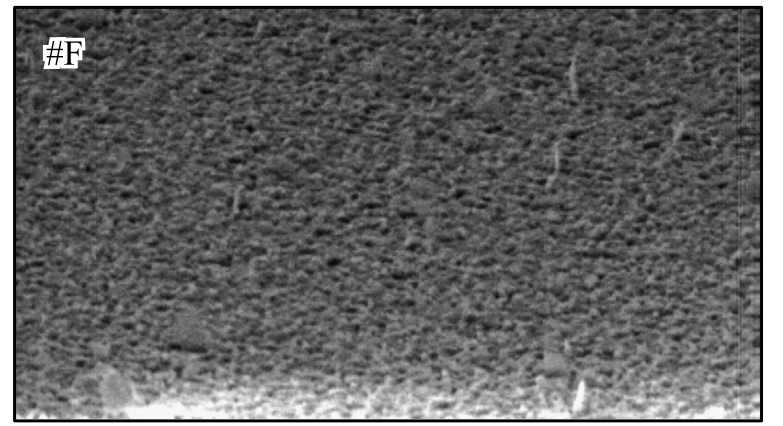

$20 \mathrm{kV}$ $b$

Рис. 2. Изображения поверхности слоев $\mathrm{GaSe}$, выращенных на подложках $\mathrm{GaAs}(001)$ и $\mathrm{GaAs}(112)$ при различных условиях, полученные методом РЭМ.

$\operatorname{GaAs}(001)$ при $T_{s}=400^{\circ} \mathrm{C}$. Хорошо видно, что слой содержит большую плотность структурных дефектов, что во многом обусловлено многодоменной структурой пленки. Картины электронной дифракции показывают, что выращенный слой $\mathrm{GaSe}$ имеет преимущественно ромбоэдрическую структуру, что соответствует политипу $\gamma$-GaSe. Формирование политипа $\gamma$-GaSe ранее наблюдалось при МПЭ $\mathrm{GaSe}$ на подложках из $\mathrm{GaAs}(001)$ и слюды $[6,10,14]$. На изображении ПЭМ высокого разрешения (рис. $3, b$ ) отчетливо наблюдается слоистая структура выращенной пленки, при этом период решетки в вертикальном направлении составляет $\sim 0.8 \mathrm{Hм}$, что соответствует толщине одного тетраслоя GaSe. Направление оси с слоя совпадает с направлением нормали к поверхности роста. Тем не менее уже на начальных стадиях эпитаксии наблюдается разориентация доменов относительно друг друга на углы до $5^{\circ}$ (рис. $3, b$ ), которая может быть частично вызвана изначальной шероховатостью поверхности подложки $\mathrm{GaAs}(001)$ после отжига в вакууме в отсутствие потока As.

На рис. 4 приведен спектр КРС „объемного“ слоя GaSe производства HQ-graphene (кривая 1). В спектре наблюдаются линии на 19, 59, 134, 211, 214 и $307.5 \mathrm{~cm}^{-1}$. Эти частоты очень близки к значениям, приводимым для колебательных мод симметрии $E^{\prime}\left(1^{2}\right), E^{\prime \prime}\left(1^{2}\right), A^{\prime}\left(1^{2}\right)$, $E^{\prime \prime}\left(2^{2}\right), E^{\prime}\left(2^{2}\right) \mathrm{TO}, A_{1}^{\prime}\left(2^{2}\right)$ политипа $\varepsilon$-GaSe [15]. На этом же рисунке представлены типичные спектры КРС слоев $\mathrm{GaSe}$, выращенных на подложках $\mathrm{GaAs}(001)$ (кривая 2, образец \#В) и $\operatorname{GaAs}(112)$ (кривая 3, образец \#D). B спектрах этих образцов наблюдаются линии, соответствующие колебательным модам в $\mathrm{GaSe}$, и две линии на частотах 268 и $291 \mathrm{~cm}^{-1}$, связанные с рассеянием 

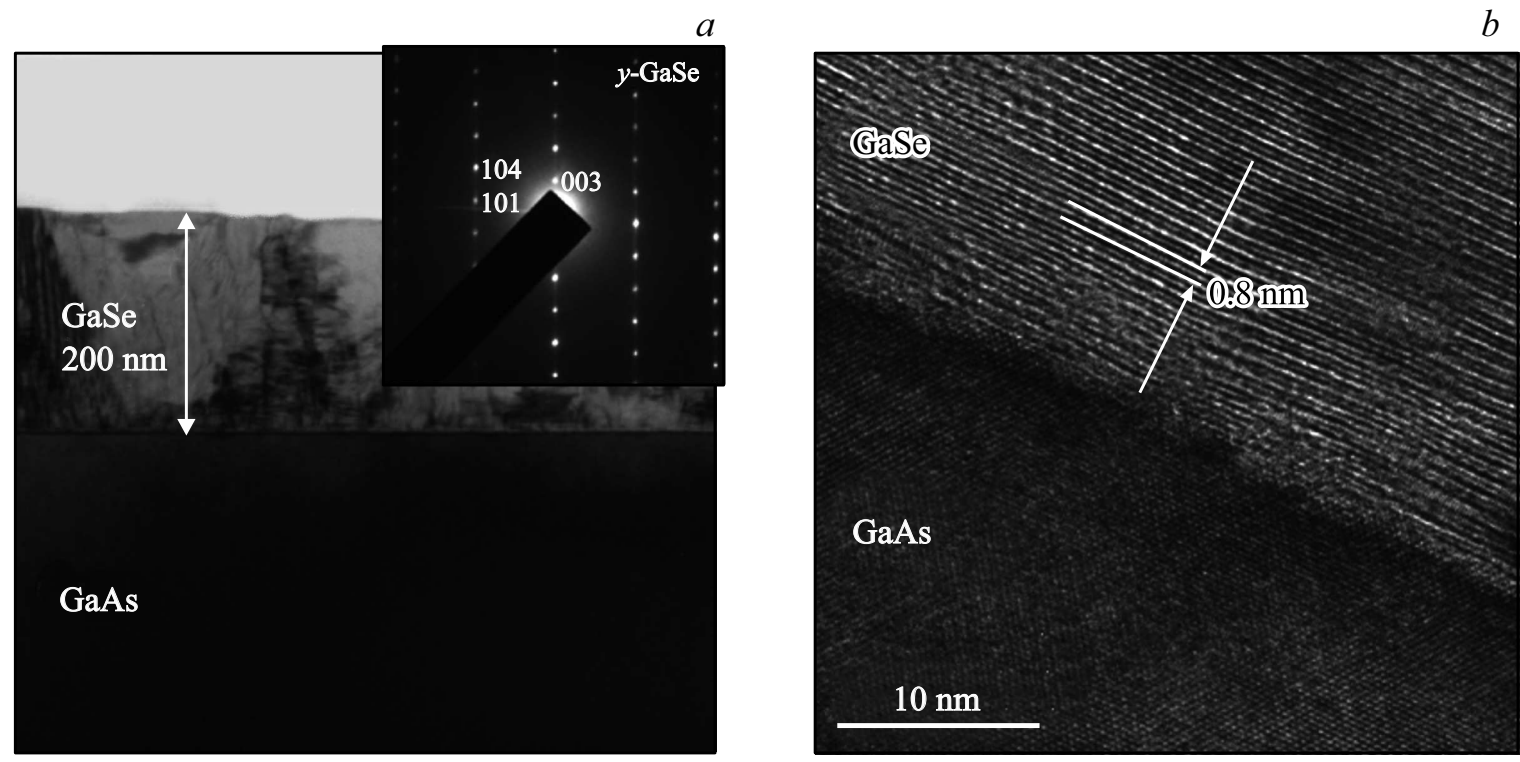

Рис. 3. Изображения структуры \#В в геометрии поперечного сечения, полученные методом ПЭМ $(a)$ и ПЭМ высокого разрешения $(b)$. a: на вставке - картина электронной дифракции структуры \#B, показывающая, что выращенный слой GaSe имеет преимущественно ромбоэдрическую структуру, соответствующую политипу $\gamma$-GaSe.

света на TO- и LO-фононах в используемых подложках GaAs. Видно, что только две линии в спектрах КРС пленок GaSe, а именно на частотах 59 и $308 \mathrm{~cm}^{-1}$, очень близки по своим значениям к соответствующим линиям спектра „объемного“ слоя $\varepsilon-\mathrm{GaSe}$. Две оставшиеся линии, регистрируемые на частотах 132 и $206 \mathrm{~cm}^{-1}$, имеют низкочастотный сдвиг по сравнению с аналогичными линиями в спектре „объемного“ $\varepsilon$-GaSe, которые регистрируются на частотах 134 и $214 \mathrm{~cm}^{-1}$. В работе [15] приводятся спектры КРС монокристалла политипа $\mathcal{E}$-GaSe, полученного методом Бриджмена, и спектры КРС политипа $\gamma$-GaSe, измеренные на игольчатых кристаллах, полученных методом вакуумной возгонки. Анализ данных, представленных в этой работе, приводит к заключению, что отмеченные выше особенности в поведении фононных линий в спектрах КРС выращенных эпитаксиальных пленок GaSe характерны для политипа $\gamma$-GaSe. Дополнительным аргументом в пользу такого утверждения является полное отсутствие в спектрах КРС эпитаксиальных пленок GaSe линии на частоте $19 \mathrm{~cm}^{-1}$, которая должна соответствовать межплоскостным колебаниям. В работе [16] было показано, что элементарная ячейка политипа $\gamma$-GaSe, описываемого пространственной группой симметрии $C_{3 v}^{5}$, содержит только один слой. Следовательно, характерной особенностью такого политипа должно быть отсутствие межплоскостных колебаний. В этой же работе приводится низкочастотный спектр КРС политипа $\gamma$-GaSe, в котором так же, как в спектрах эпитаксиальных пленок GaSe, представленных на рис. 4, наблюдается только линия на частоте $59 \mathrm{~cm}^{-1}$ и полностью отсутствует линия на частоте $19 \mathrm{~cm}^{-1}$. Положение основных линий в спектрах

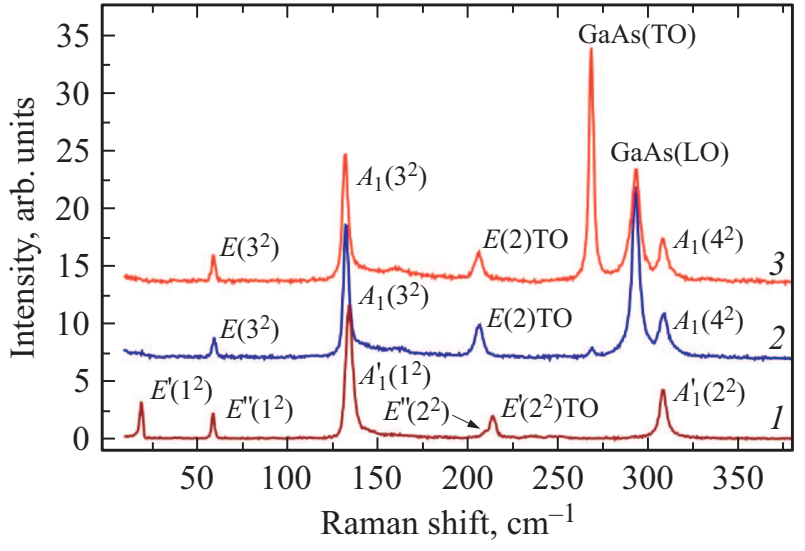

Рис. 4. Спектр КРС „объемного“ слоя $\mathcal{\varepsilon}$-GaSe производства HQ-graphene (1) и типичные спектры КРС слоев GaSe на примере слоев \#В на подложке $\mathrm{GaAs}(001)$ (2) и \#D на подложке $\operatorname{GaAs}(112)$ (3). Все спектры нормированы на интенсивность линий симметрии $A_{1}^{\prime}\left(1^{2}\right)$ и $A_{1}\left(3^{2}\right)$. Длина волны возбуждения 532 нм, мощность возбуждения 80 мкВт, температура измерений $293 \mathrm{~K}$.

КРС выращенных слоев хорошо согласуется с опубликованным ранее спектром КРС для эпитаксиального слоя $\gamma$-GaSe [14], при этом в [14] соответствие выращенного слоя политипу $\gamma$-GaSe было также определено методом электронной дифракции. Также необходимо отметить, что в спектрах КРС всех выращенных образцов отсутствуют какие-либо особенности, которые можно было бы соотнести с наличием включений фазы $\mathrm{Ga}_{2} \mathrm{Se}_{3}$.

На рис. 5 приведены кривые РД слоев GaSe \#A, \#B, \#Е и \#F, выращенных на подложках $\mathrm{GaAs}(001)$. На кривых 


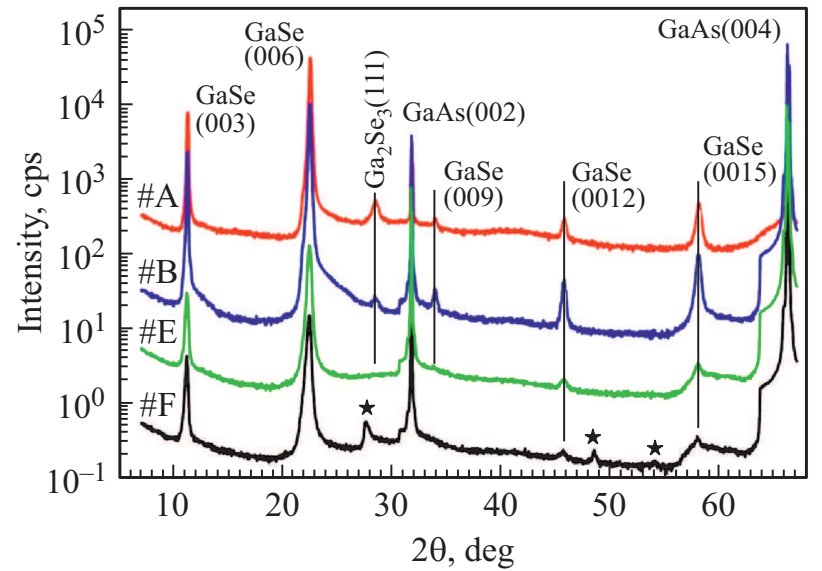

Рис. 5. Кривые РД слоев GaSe \#A, \#В, \#Е и \#F, выращенных на подложках $\operatorname{GaAs}(001)$. Плоскости индицированы для ромбоэдрической фазы $\gamma$-GaSe. В слоях \#А и \#В (две верхних кривых), выращенных в условиях сильного обогащения поверхности роста селеном, присутствует пик, который может быть атрибутирован как отражение от плоскости $\mathrm{Ga}_{2} \mathrm{Se}_{3}(111)$.

РД помимо отражений от плоскостей (002) и (004) подложки GaAs наблюдаются отражения от плоскостей $\mathrm{GaSe}$, перпендикулярных оси с, что указывает на преимущественную ориентацию оси с в направлении нормали к поверхности роста. На кривых РД слоев \#А и \#В, выращенных в условиях сильного обогащения поверхности роста селеном, присутствует дополнительный пик в области углов $\sim 28.4^{\circ}$ по оси $2 \theta$, который указывает на наличие включений фазы $\mathrm{Ga}_{2} \mathrm{Se}_{3}$, так как его положение хорошо согласуется с отражением от плоскости $\alpha-\mathrm{Ga}_{2} \mathrm{Se}_{3}(111)$ [17]. Необходимо отметить, что в [12] этот пик, доминирующий в кривых РД слоев $\mathrm{GaSe}$, выращенных методом МПЭ в условиях сильного пересыщения по Se, был также атрибутирован образованием „паразитной“ фазы $\mathrm{Ga}_{2} \mathrm{Se}_{3}$. В слоях $\mathrm{GaSe}$, выращенных в условиях вблизи стехиометрии на поверхности роста, пик $\mathrm{Ga}_{2} \mathrm{Se}_{3}(111)$ отсутствует (две нижние кривые на рис. 4). Преимущественная ориентация фазы $\alpha-\mathrm{Ga}_{2} \mathrm{Se}_{3}$ обусловлена близостью расстояний между атомами в плоскостях $\mathrm{GaSe}(0001)$ и $\mathrm{Ga}_{2} \mathrm{Se}_{3}(111)$ (т.е. вдоль направлений $\mathrm{GaSe}\langle 1000\rangle$ и $\left.\mathrm{Ga}_{2} \mathrm{Se}_{3}\langle 110\rangle\right)-3.755$ и $3.83 \AA$ соответственно. Согласно [17], период решетки кубического $\alpha-\mathrm{Ga}_{2} \mathrm{Se}_{3}$, определенный из анализа кривых РД, составляет $a=5.44 \AA$.

На кривой РД слоя \#F, выращенного с высокой скоростью, $\sim 5$ нм/мин, при низкой температуре $\left(T_{s}=400^{\circ} \mathrm{C}\right)$ и обладающего разветвленной поверхностью, наблюдаются дополнительные пики (отмечены звездочками), которые могут быть интерпретированы как отражения от наклонных плоскостей GaSe. Появление этих дополнительных рефлексов на кривой РД, по-видимому, является отражением внутреннего упорядочения, или, другими словами, существования преимущественных углов наклона нанопластинок в структуре \#F, а отсутствие каких-либо дополнительных особенностей на кривой РД слоя \#Е, вероятнее всего, обусловлено его меньшей толщиной по сравнению с образцами \#А, \#В и \#F.

\section{4. Заключение}

В работе исследованы слои слоистого GaSe, полученные методом МПЭ при температуре $T_{s}=400-500^{\circ} \mathrm{C}$ на подложках $\operatorname{GaAs}(001)$ и $\mathrm{GaAs}(112)$ при использовании элементного источника $\mathrm{Ga}$ и клапанного источника $\mathrm{Se}$ с температурой зоны разложения $T_{\mathrm{Se}}(\mathrm{cr})=500^{\circ} \mathrm{C}$. Мeтодами электронной дифракции и комбинационного рассеяния света установлено, что выращенные слои $\mathrm{GaSe}$ соответствуют политипу $\gamma$-GaSe. Ось с в слоях GaSe, выращенных на подложках $\mathrm{GaAs}(001)$ при $T_{s}=400^{\circ} \mathrm{C}$, направлена по нормали к поверхности роста, что подтверждается ПЭМ-изображениями в геометрии поперечного сечения и анализом кривых РД. Определены основные факторы, влияющие на морфологию выращиваемых 2D слоев. Показано, что поверхность становится более планарной при приближении условий роста к стехиометрическим, при этом условию стехиометрии на поверхности роста при $T_{\mathrm{Se}}(\mathrm{cr})=500$ и $400^{\circ} \mathrm{C}$ отвечает отношение падающих потоков $P_{\mathrm{Se}} / P_{\mathrm{Ga}}(\mathrm{BEP}) \approx 12$. Важнейшим фактором, влияющим на морфологию растущего слоя GaSe при низких температурах эпитаксиального роста $\left(T_{s}=400^{\circ} \mathrm{C}\right)$, является скорость осаждения $\mathrm{GaSe}$, определяемая потоком атомов $\mathrm{Ga}$. При высоких значениях потока $\mathrm{Ga}$ (соответствующих скорости роста $\mathrm{GaSe}$ $\sim 4-5$ нм/мин) низкая подвижность атомов $\mathrm{Ga}$ приводит к увеличению шероховатости поверхности растущего слоя. Спектры РД показывают, что в слоях GaSe, выращенных в условиях сильного обогащения поверхности роста селеном, присутствуют включения „паразитной“ фазы $\mathrm{Ga}_{2} \mathrm{Se}_{3}$.

\section{Благодарности}

РД-исследования и ПЭМ-измерения выполнены с использованием оборудования федерального ЦКП „Материаловедение и диагностика в передовых технологиях“ при частичной финансовой поддержке Министерства образования и науки РФ (идентификатор проекта RFMEFI62117X0018).

\section{Конфликт интересов}

Авторы заявляют, что у них нет конфликта интересов.

\section{Список литературы}

[1] A. Kuhn, A. Chevy, R. Chevalier. Phys. Status Solidi A, 31, 469 (1975).

[2] T.E. Beechem, B.M. Kowalski, M.T. Brumbach, A.E. McDonald, C.D. Spataru, S.W. Howell, T. Ohta, J.A. Pask, N.G. Kalugin. Appl. Phys. Lett., 107, 173103 (2015). 
[3] J. Susoma, J. Lahtinen, M. Kim, J. Riikonen, H. Lipsanen. AIP Advances, 7, 015014 (2017).

[4] W. Choi, N. Choudhary, G.H. Han, J. Park, D. Akinwande, Y.H. Lee. Materials Today, 20 (3), 116 (2017).

[5] N. Kojima, K. Sato, A. Yamada, M. Konagai, K. Takahashi. Jpn. J. Appl. Phys., 33, L1482 (1994).

[6] Z.R. Dai, S.R. Chegwidden, L.E. Rumaner, F.S. Ohuchi. J. Appl. Phys., 85, 2603 (1999).

[7] M. Budiman, T. Okamoto, A. Yamada, M. Konagai. Appl. Surf. Sci., 117/118, 518 (1997).

[8] M. Budiman, T. Okamoto, A. Yamada, M. Konagai. Jpn. J. Appl. Phys., 37, 5497 (1998).

[9] A. Koma. J. Cryst. Growth, 201/202, 236 (1999).

[10] N. Kojima, K. Sato, M. Budiman, A. Yamada, M. Konagai, K. Takahashi, Y. Nakamura, O. Nittono. J. Cryst. Growth, 150, 1175 (1995).

[11] D.J. O’Hara, T. Zhu, A.H. Trout, A.S. Ahmed, Y.K. Luo, C.H. Lee, M.R. Brenner, S. Rajan, J.A. Gupta, D.W. McComb, R.K. Kawakami. Nano Lett., 18 (5), 3125 (2018).

[12] C.H. Lee, S. Krishnamoorthy, D.J. O'Hara, M.R. Brenner, J.M. Johnson, J.S. Jamison, R.C. Myers, R.K. Kawakami, J. Hwang, S. Rajan. J. Appl. Phys., 121, 094302 (2017).

[13] S.V. Ivanov, S.V. Sorokin, I.V. Sedova. In: Molecular Beam Epitaxy: From research to mass production, ed. by M. Henini, 2nd edn (Elsevier Inc., 2018) p. 571.

[14] X. Yuan, L. Tang, Sh. Liu, P. Wang, Zh. Chen, Ch. Zhang, Y. Liu, W. Wang, Y. Zou, C. Liu, N. Guo, J. Zou, P. Zhou, W. Hu, F. Xiu. Nano Lett., 15, 3571 (2015).

[15] R.M. Hoff, J.C. Irwin, R.M.A. Lieth. Can. J. Phys., 53, 1606 (1975).

[16] A. Polian, K. Kunc, A. Kuhn. Solid State Commun., 19, 1079 (1976).

[17] M.A. Afifi, A.E. Bekheet, H.T. El-Shair, I.T. Zedan. Physica B, 325, 308 (2003).

Редактор Л.В. Шаронова

\title{
Molecular beam epitaxy of 2D layered GaSe grown on GaAs(001) and GaAs(112) substrates: structural and optical properties
}

\author{
S.V. Sorokin, P.S. Avdienko, I.V. Sedova, \\ D.A. Kirilenko, M.A. Yagovkina, A.N. Smirnov, \\ V.Yu. Davydov, S.V. Ivanov \\ loffe Institute, \\ 194021 St. Petersburg, Russia
}

\footnotetext{
Abstract We present the study of structural and optical properties of 2D GaSe layers grown by molecular beam epitaxy (MBE) on $\mathrm{GaAs}(001)$ and $\mathrm{GaAs}(112)$ substrates using valve cracking cell as a Se source. The influence of the MBE growth parameters (substrate temperature, Ga flux intensity, Se/Ga flux ratio) on the surface morphology of GaSe layers has been studied in detail. Using transmission electron microscopy, electron diffraction and Raman spectroscopy techniques it has been established that the GaSe layers possess the lattice of $\gamma$-polytype. X-ray diffraction has revealed presence of inclusions of $\alpha-\mathrm{Ga}_{2} \mathrm{Se}_{3}$ phase in GaSe layers grown under strong Se-rich conditions.
} 